\title{
Torque de Retiro de Implantes de Titanio Instalados en Tibia de Perros con Defecto Óseo
}

\author{
The Removal Torque of Titanium Implant Inserted in Dog Tibia with a Bone Defect
}

\author{
María de Almeida*; Antonio Lanata-Flores*; Sergio Olate ${ }^{* * * * * *}$; Leandro Pozzer*; \\ Mario Cantín $^{* * *}$; Bélgica Vásquez ${ }^{* * * *}$ \& José de Albergaria-Barbosa*
}

\begin{abstract}
ALMEIDA, M.; LANATA-FLORES, A.; OLATE, S.; POZZER, L.; CANTÍN, M.; VÁSQUEZ, B. \& ALBERGARIA-BARBOSA, J. Removal torque of titanium implant inserted in dog tibia with a bone defect. Int. J. Morphol., 31(2):700-705, 2013.

RESUMEN: El torque de remoción es utilizado para estudiar el comportamiento de implantes y su oseointegración. El objetivo de esta investigación fue determinar el torque de retiro de implantes dentales con defectos óseo asociados de tipo fenestración. Seis perros (Canis familiaris) fueron en semejantes condiciones de peso y talla seleccionados para la investigación. Se realizó un acceso quirúrgico al fémur del animal sitio donde se instalaron 3 implantes, realizándose defectos circulares de $5 \mathrm{~mm}$ en cada implante. El defecto fue cubierto con membrana absorbible (colágeno), membrana no absorbible (celulosa) o sin cobertura (control). Los animales fueron sometidos a eutanasia a las 3 semanas y a los 8 semanas, momento en que se les realizó el torque de retiro a los implantes con una llave especialmente diseñada para el procedimiento; el análisis de datos se realizó con la prueba de Turkey con significancia estadística p<0.1. Se observó una creciente reparación ósea en los diferentes tiempos de eutanasia; el defecto control reveló un menor nivel de reparación al ser comparado con los defectos cubiertos por membranas. Se realizó el torque de retiro en los implantes estableciendo valores crecientes de unión hueso-implante en los tiempos de eutanasia. No existió diferencias significativas en los torques de retiro de los implantes en ninguno de los defectos generados. Finalmente se puede concluir que las membranas biológicas y la reparación no influyó en el torque de retiro de implantes dentales.
\end{abstract}

PALABRASCLAVE: Torque retiro; Oseointegración; Regeneración ósea.

\section{INTRODUCCIÓN}

La oseointegración es fundamental en la terapia con implantes dentarios y para obtenerla depende de una adecuada biocompatibilidad, calidad de superficie del implante, técnica quirúrgica, adecuado estatus del huésped y condiciones de carga (Albreksson et al., 1986).

La superficie del implante es un elemento fundamental para entender la interacción con el hueso adyacente (Buser et al., 1991, Wennerberg et al., 1996). El aumento de la rugosidad y modificaciones en la macrogeometría y la microgeometría permite estabilizar implantes en la estructura ósea (Cho et al., 2003).

Algunos desafíos clínicos se presentan cuando existen defectos que muchas veces acompañan los implantes instalados, los cuales podrían responder de forma inadecuada a la carga mecánica y la estabilidad del implante (Dung et al., 2012). En estas condiciones el uso de injertos óseos y membranas biológicas podría contribuir a mejorar la estabilidad del sistema.

Una de las formas de reconocer la estabilidad del implante instalado es a través del torque de retiro que consiste en realizar el contra-torque en el implante instalado a fin de informar el grado de estabilidad del mismo mediante el quiebre de la interface implante-hueso; esto se fundamenta en el hecho de que existe una relación establecida entre torque de retiro y el nivel de oseointegración (Johansson \& Albrektsson, 1991).

El objetivo de esta investigación es estudiar el torque de retiro de implantes dentales acompañados de defecto de tipo fenestración tratados o no con membranas biológicas.

\footnotetext{
* Division of Oral and Maxillofacial Surgery, Piracicaba Dental School, State University of Campinas, Brazil.

** Division of Oral and Maxillofacial Surgery and CIMA Group, University of La Frontera, Chile.

*** Universidad Autónoma de Chile. Centre of Research of Biomedical Sciences, Temuco, Chile.

***** Universidad de Tarapacá, Arica, Chile
} 


\section{MATERIAL Y MÉTODO}

Fueron utilizados 6 perros adultos sanos con peso aproximado de $6 \mathrm{~kg}$ obtenidos del bioterio de la Universidad Estadual de Campinas, Brasil. Los animales fueron divididos en tres grupos (grupo experimental 1, experimental 2 y control), mantenidos con alimentación sólida y agua. Previo a los procedimientos quirúrgicos, los animales fueron sedados con clorhidrato de ketamina $(0,15 \mathrm{ml} / \mathrm{kg})$, por vía intramuscular siendo sometidos a anestesia general con pentobarbital sódico al 3\% (30mg/kg). El estudio fue aprobado por el Comité de Ética en Investigación de la Universidad Estaudal de Campinas.

Procedimiento quirúrgico. Después de la fase de anestesia general, los animales fueron sometidos a tricotomía de la zona junto a técnicas de asepsia y antisepsia y posterior aislación del campo operatorio. El lugar de intervención fue la tibia del animal para lo cual se accedió con bisturí $\mathrm{N}^{\circ} 15$ con una incisión de $5 \mathrm{~cm}$ de longitud. Se realizó la divulsión y descolamiento del sector hasta liberar el periostio del tejido óseo.

Con la exposición ósea se establecieron tres lugares de perforación con $1 \mathrm{~cm}$ de diferencia entre cada uno. En cada lugar se procedió a realizar una perforación de $9 \mathrm{~mm}$ de profundidad utilizando un contrangulo 16:1 montado en motor de funcionamiento a $1000 \mathrm{rpm}$; la perforación se realizó con abundante irrigación de suero fisiológico. La secuencia de fresas fue utilizada según las indicaciones del fabricante (INP, Sistema de Implantes Nacionais e de Próteses Comércio Ltda.).

Previo a la instalación del implante y cuando ya se había construido el lecho de instalación del mismo se confeccionaron defectos óseos en cada lugar mediante la instrumentación con una trefina de $5 \mathrm{~mm}$ de diámetro generando defectos circulares en la fase medial de la tibia que asemejaran los defectos de tipo fenestración presentes en la instalación de algunos implantes en maxilares edéntulos. Luego de esta etapa, profusa irrigación y limpieza fue realizada para eliminar los detritus de la preparación.

Posteriormente, en cada perforación se instaló un implante de titanio de $9 \mathrm{~mm}$ de largo y 3,5 $\mathrm{mm}$ de ancho, conexión de hexágono externo, con superficie rugosa debido al tratamiento químico-mecánico con arenado realizado por el fabricante. El implante fue retirado de su envoltura y fue instalado en el sitio según las indicaciones del fabricante confirmando sobe $35 \mathrm{~N}$ de torque de instalación con el uso de llaves de catraca especialmente diseñadas para esta fase. Dos de los tres defectos de $5 \mathrm{~mm}$ fueron cubiertos con mem- branas mientras que el tercero fue mantenido sin protección de membrana para utilizarlo como control. Las membranas fueron instaladas sobre el defecto considerando al menos 5 $\mathrm{mm}$ de contacto entre membrana y hueso para estabilizar la posición de las membranas; conjuntamente las membranas fueron hidratadas con suero fisiológico para hacerlas modelables.

Selección de membranas utilizadas. Se realizaron tres defectos en los tres implantes donde se instalaron membrana en dos de ellos, seleccionando de forma aleatoria la instalación de membrana absorbible (grupo experimental 1), no absorbible (grupo experimental 2) y un defecto sin membranas (grupo control). Las características de la membrana fueron:

Membrana no absorbible: se utilizó una membrana de celulosa fabricada a partir de piel artificial. Consiste en una matriz biosintética de dos capas biológicamente inerte, semipermeable, semi-transparente y resistente a la tracción. La capa interna está constituida por una red de microfibrillas de celulosa cristalina que le entrega la rigidez a la membrana; la capa externa está formada de alcalicelulosa con porosidad variable.

Membrana absorbible: corresponde a una membrana de colágeno con matriz no friable elaborada del tendón flexor bovino; presenta una forma de superficie plana, condensada y texturizada con poros de $0,004 \mathrm{~mm}$

Eutanasia y análisis de torque. Se estimaron periodos de eutanasia de tres animales a las tres semanas y tres animales a las ocho semanas desde la primera cirugía. Para el periodo de eutanasia se utilizó el mismo esquema farmacológico indicado previamente y el procedimiento para exponer los implantes y el defecto siguió los mismos pasos que la primera cirugía.

El torque de retiro del implante se realizó con una llave especialmente diseñada para ingresar en el hexágono conector del implante aumentando la unidad de torque gradualmente hasta quebrar la interface entre hueso y dispositivo; los animales fueron sometidos a eutanasia con una dosis letal de cloruro de potasio al 19,1\%. El análisis de datos se realizó con la prueba ANOVA para estudio de grupos y se utilizó la prueba estadística de test $\mathrm{F}$ con nivel de significancia en $1 \%$.

Procesamiento de muestras y análisis histológico. Para exponer los implantes y defectos, se siguieron los mismos pasos que la primera cirugía. Las muestras de bloques óseos fueron removidos desde la tibia utilizando un margen de seguridad $1 \mathrm{~mm}$ lateral a los implantes instalados. Las mues- 


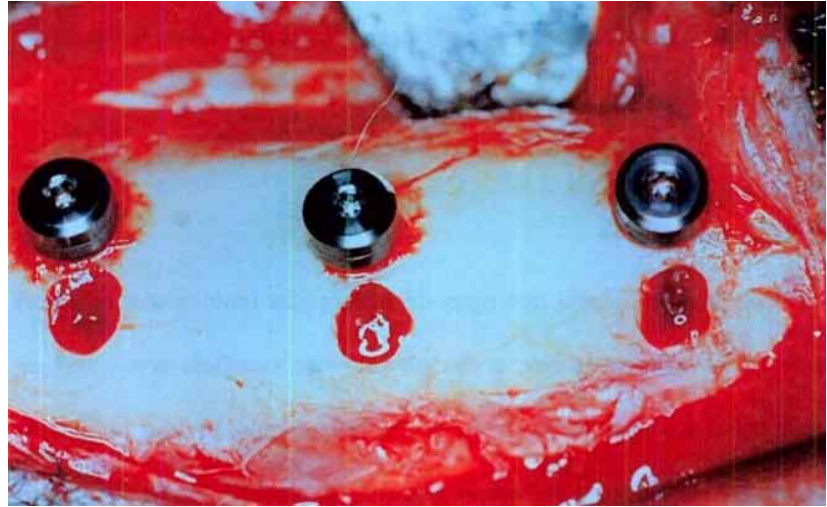

Fig. 1. Modelo animal con los implantes dentales instalados junto a los defectos generados.

tras fueron fijadas en formaldehído al $4 \%$ durante $48 \mathrm{~h}$ a temperatura ambiente, lavadas con agua y descalcificadas en solución de citrato de sodio al $20 \%$ y acido fórmico al $50 \%$ en partes iguales durante siete meses. Cada bloque óseo fue seccionado longitudinalmente al implante, incluyendo la zona del defecto artificial, y se separó el hueso del implante. Las muestras óseas fueron deshidratadas en soluciones decrecientes de alcohol etílico e incluidas en parafina para su análisis histológico.

Se prepararon secciones histológicas seriadas de 6 $\mathrm{mm}$ de grosor, $\mathrm{y}$ fueron teñidas siguiendo los protocolos para técnicas de hematoxilina-eosina y tricromio de Mallory, y observación a través de microscopio óptico. El análisis descriptivo definió la presencia de vasos sanguíneos, células óseas, trabéculas, espacios medulares y tejido conectivo, con aumentos de 10X y 100X.

\section{RESULTADOS}

\section{Análisis grupo con membrana absorbible}

Tres semanas. Se observó actividad osteogénica en la proximidad del periostio junto a una formación ósea incipiente. En el defecto completo se observó trabeculado óseo de aspecto inmaduro, manteniendo clara diferencia entre éste y el hueso adyacente que presentaba un patrón compacto con canales de harvers y láminas concéntricas. La neoformación ósea parecía inicial desde la región endosteal hacia el interior del defecto; no se observó áreas de reabsorción o células inflamatorias

Ocho semanas. Los defectos se observaron totalmente rellenos de tejido óseo neoformado con características de mayor maduración, un patrón óseo más denso y espacios

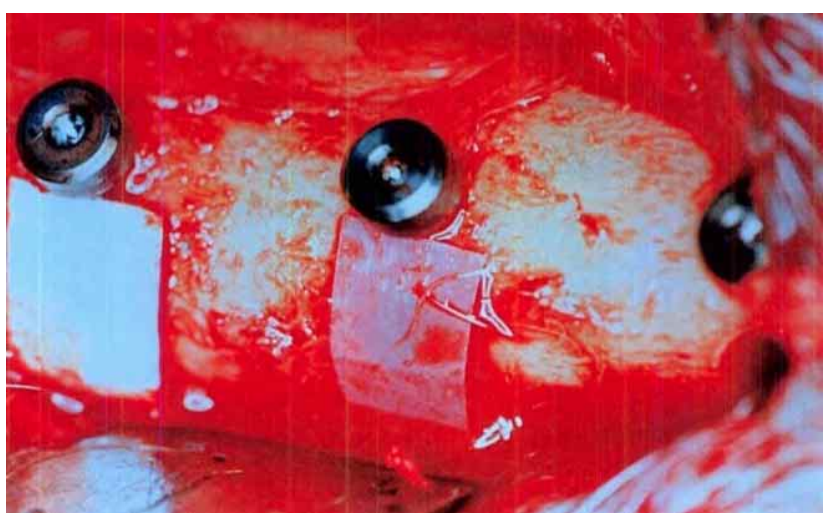

Fig. 2. Membranas instaladas de acuerdo a la organización de grupos instalando las membranas no absorbibles, absorbibles y defecto sin membrana (control).

medulares reducidos; aún fue observada la presencia de osteoblastos y vasos sanguíneos. En algunos lugares no se diferencia entre el área de neoformación ósea y el hueso preexistente (Fig. 3).

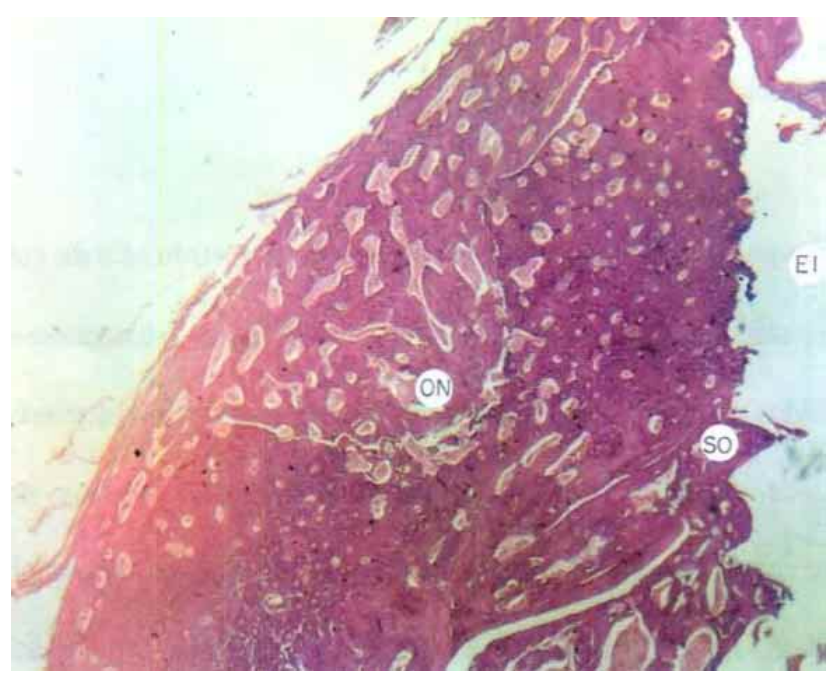

Fig. 3. Defecto cubierto por membrana absorbible, ocho semanas. Se observa alto nivel de neoformación ósea con ausencia de la membrana gracias a la reabsorción de la misma. En lugares no se identifica la diferencia entre segmentos nativo y regenerado presentando un mayor trabeculado óseo que sus pares.

\section{Análisis grupo con membrana absorbible}

Tres semanas. Se observó actividad osteoblástica con neoformación ósea que rellena el defecto, hueso inmaduro y algunos vasos sanguíneos. Se presentó un claro límite entre el defecto y el hueso adyacente, sin presencia de osteoclastos o infiltrado inflamatorio.

Ocho semanas. Los defectos óseos se observaron rellenos con nuevo hueso formado, presentando espacios medulares reducidos y trabéculas de aspecto compacto. En algunas 
imágenes se observó la membrana de celulosa en contacto con tejido conectivo; se observó aposición ósea de tejido neoformado en los márgenes del defecto sin evidencias de áreas de necrosis. La actividad osteoclástica y el infiltrado inflamatorio estaban ausentes (Fig. 4).

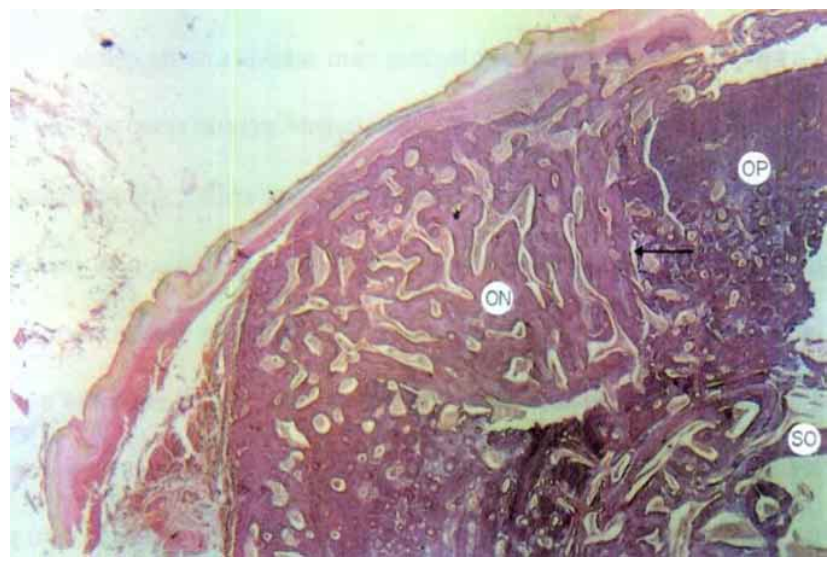

Fig. 4. Defecto cubierto por membrana no absorbible, ocho semanas. Se observa la membrana presente con tejido conectivo cercano a la membrana. Presenta un elevado nivel de reparación ósea con limitadas diferencias entre el hueso nativo y el regenerado.

\section{Análisis grupo sin protección de membrana}

Tres semanas. Se observó presencia de tejido óseo inmaduro al interior del defecto con amplios espacios medulares y trabéculas, osteoblastos en la periferia, tejido conectivo y vasos sanguíneos. Se presentó menor cantidad de tejido óseo en formación al comparar con los defectos cubiertos por membrana; los márgenes entre el defecto y hueso adyacente fueron bien diferenciados.

Grupo control ocho semanas. Se observaron múltiples áreas de tejido fibroso sin indicios de actividad osteoblástica. En la mayoría de las láminas se observó tejido óseo aislado e inmaduro con invaginación del tejido conectivo hacia el interior del defecto. El tejido óseo de neoformación se observó en la periferia del defecto, con claras diferencias respecto al hueso preexistente. (Fig. 5).

Análisis de Torque de Retiro. El torque utilizado para el rompimiento de interface de los implantes no fue influenciado por el tipo de membrana utilizada o por la au-

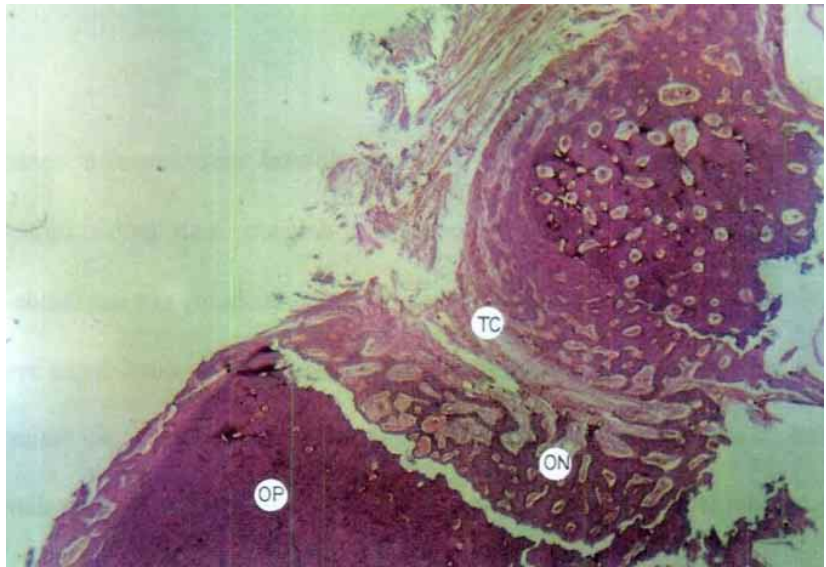

Fig. 5. Defecto no cubierto por membranas, ocho semanas. Se observa un total colapso y el ingreso de tejido conectivo al interior de la cavidad. Existe deficiencias en la formación ósea con importante tejido conectivo.

sencia de la misma; sin embargo, el periodo de eutanasia y reparación ósea perimplantar influenció en la fuerza aplicada para conseguir el quiebre de la interface implante-hueso $(\mathrm{p}<0.1)$. De esta forma, el tiempo de oseointegración es capaz de influenciar en el aumento del torque necesario para retiro del implantes mientras que las membranas biológicas no presentan influencias al respecto (Tabla I). El torque de retiro fue mayor en el periodo de ocho semanas que en el periodo de tres semanas; sin embargo no se observó diferencias en el torque de retiro del implante en ninguna de las situaciones exploradas.

\section{DISCUSION}

La reparación ósea es influenciada por diversos factores tales como el tamaño y forma del defecto, calidad ósea cortical o esponjosa, calidad del periostio adyacente, condiciones sistémicas generales del sujeto y otras (Oliveira et al., 2013). Diferentes publicaciones han señalado la dificultad de reparación ósea cuando los defectos son de tamaño críticos (Chaves Netto et al., 2009) o la capacidad vascular del tejido óseo periférico es deficiente, circunstancias en que la contribución de elementos como las membranas biológicas e injertos óseos o sustitutos pueden mejorar las condiciones de reparación.

Tabla I. Medidas de volumen óseo formado en los diferentes periodos de eutanasia $\left(\mathrm{mm}^{3}\right)$

\begin{tabular}{lcccc}
\hline \multicolumn{1}{c}{ Defecto } & 3 sem. (N cm) & P 3 sem. & 8 sem. (N cm) & P 8 sem. \\
\hline Defecto con membrana absorbible (colágeno) & 9,25 & $\mathrm{P}>0,1$ & 14,52 & $\mathrm{P}>0,1$ \\
Defecto con membrana no absorbible (celulosa) & 9,25 & $\mathrm{P}>0,1$ & 14,83 & $\mathrm{P}>0,1$ \\
Defecto control & 9,21 & $\mathrm{P}>0,1$ & 15,33 & $\mathrm{P}>0,1$ \\
\hline
\end{tabular}

Aplicación del test de Turkey con significancia estadística si $\mathrm{P}<0,1$ 
El torque de retiro se asocia al nivel de oseointegración obtenido por los implantes (Johansson \& Albrektsson 1991) de forma que es posible relacionar la regeneración ósea en diferentes defectos en relación a la unión hueso-implante que podría retener con mayor estabilidad el implante dental en determinado momento.

Cho et al. (2003) Realizaron una investigación en 10 conejos evaluando torque de retiro 12 semanas posteriores a la instalación señalando que aquellos implantes con mayor porcentaje de inclusión de "hydrofluoric acid" en su superficie presentaban un mayor torque de retiro variando desde $15 \mathrm{Ncm}$ hasta $38 \mathrm{Ncm}$.

La rugosidad de la superficie puede explicar la mayor angiogénesis, mayor alineamiento celular y finalmente mayor actividad celular (Rich, 1981; Boyan, 1996). Esto se traduce en mayor resistencia a la compresión, tensión y estrés (Thomas et al., 1987). Otros resultados revelan que los implantes maquinados presentan cerca de 25,28 $\mathrm{Ncm}$, los grit-blasted $29.57 \mathrm{~N} \mathrm{~cm}$ y los con plasmasprayed implants 40,85 $\mathrm{N} \mathrm{cm}$ (Cordioli et al.), lo cual confirma que el tratamiento de la superficie aumenta la superficie de unión con hueso promoviendo la resistencia a la tracción. Superficies tratadas con láser también han demostrado mayor torque de remoción al compararlo con otros sistemas de superficie (Cho \& Jung 2003)

Eom et al. (2012) observaron que el torque de retiro fue aumentando al pasar del tiempo y confirmó que cuando aumenta el porcentaje de contacto entre hueso e implante existe un aumento del torque necesario para retirar el implante.

La superficie, las micro-roscas y la morfología del dispositivo son relevantes en el torque de retiro aunque la publicación de Kwon et al. (2013) no presento diferencias significativas entre los diferentes macrogeometrias de implantes evaluados; en el estudio de Kwon et al., fueron relacionados con el contacto hueso-implante que tampoco presento diferencias significativas. Nuestros resultados demuestran que indistintamente del defecto óseo creado, el tratamiento del mismo (cobertura con membranas o control) no influye en al torque de retiro, lo cual nos entregas nuevas condiciones de trabajo.

Esta falta de diferencias se puede deber a que la integración de los implantes se produce en los lugares donde hay contacto con hueso y no en lugares con déficit, de forma que los tiempos utilizados en la eutanasia impiden la formación ósea que permitan integración hueso-implante o actuación en la traba mecánica del sistema (Oh et al., 2001).

Aunque la formación ósea pueda ser mayor en sectores con protección con membranas biológicas (Kim et $a l ., 2010)$, estas no serian capaces de influenciar en el torque de remoción del implante.

De esta forma, condiciones de la morfología del implante o las características de la superficie del implante serian mas importantes en la estabilidad del dispositivo en los primeros meses de instalación.

Considerando las limitaciones de este estudio, podemos concluir que la regeneración del defecto de tipo fenestración protegido por membranas no interviene en el torque de retiro. Es posible que evaluaciones del torque de retiro a mayor plazo entreguen diferencias en base al tiempo necesario para obtener reparación ósea del sitio tratado.

ALMEIDA, M.; LANATA-FLORES, A.; OLATE, S.; POZZER, L.; CANTÍN, M.; VÁSQUEZ, B. \& ALBERGARIA-BARBOSA, J. Removal torque of titanium implant inserted in dog tibia with a bone defect. Int. J. Morphol., 31(2):700-705, 2013.

SUMMARY: The removal torque has been used for analysis of implant and their osseointegration. The aim of this research was to determine the removal torque of dental implants installed with fenestration-type bone defect. Six dogs with similar conditions of weight and height were selected for this research. We realized a surgical approach to the tibia and in place installed 3 dental implants with $5 \mathrm{~mm}$ - diameter circular bone defect in each implant. The bone defects were covered with absorbable membrane (collagen), non absorbable membrane (cellulose) and one (control) defect without coverage. The animals were euthanized at three weeks and at eight weeks when torque withdrawal of the implant was realized with a ratchet especially designed for this procedure. Data analyses was realized with Turkey test with statistical significance of $\mathrm{p}<0.1$. We observed an increasing bone repair for the two periods of euthanasia; the control defect show minor level of reparation when compared to defect coverage for membrane. We realized the removal torque with increasing values of bone-implant union for two periods of euthanasia. No difference was observed in removal torque in either defect with or without membrane. Finally, we conclude that biological membrane and bone repair was not influenced in the removal torque of dental implants.

KEY WORD: Removal torque; Osseointegration; Bone regeneration. 


\section{REFERENCIAS BIBLIOGRÁFICAS}

Albreksson, T.; Zarb, G. \& Eriksson, A. R. The long-term efficacy of currently used dental implant; A review and proposed criteria of success. Int. J. Oral. Maxillofac. Implants, 1:11-5, 1986.

Buser, D.; Schenk, R. \& Steinemann, S. Influence of surface characteristics on bone integration of titanium implants. A histomophometric study in minature pigs. J. Biomed. Mater. Res., 25:9-902, 1991.

Boyan, B. D.; Hummert, T. W.; Dean, T. T. \& Schwartz. Role of material surfaces in regulation bone and cartilage cell response. Biomaterials, 17:137-46, 1996.

Cordioli, G.; Majzoub, Z.; Piatelli, A. \& Scarano, A. Removal torque and histomorphometric investigation of 4 different titanium surfaces. Int. J. Oral Maxillofac. Implants, 15:66874, 2000.

Chaves Netto, H. D. M.; Olate, S.; Chaves, M. M. G. A.; Barbosa, A. J. R. \& Mazzonetto, R. Histological analyses of osseous repair defetcs. Recognized of critic defects. Int. J. Morphol., 27:1121-7, 2009.

Cho, S. A. \& Park, K. T. The removal torque of titanium screw inserted in rabbit tibia treated by dual acid etching. Biomaterials, 24:3611-7, 2003.

Cho, S. A. \& Jung, S. K. A removal toriqye of the laser-treated titanium implants in rabbit tibia. Biomaterials, 24: 4859-63, 2003.

Eom, T. G.; Jeon, G. R.; Jeong, C. M.; Kim, Y. K.; Kim, S. G.; Cho, I. H.; Cheok, Y. S. \& Oh, J. S. Experimental study of bone response to hydroxiapatite implants: bone-implant contact and removal torque test. Oral Surg. Oral Med. Oral Pathol. Oral Radiol., 114:411-8, 2012.

Johansson, C. B. \& Albrektsson, T. A removal torque and historphometric study of commercially pure niobium and titanium implants in rabbit bone. Clin. Oral Implant Res., 2:24-9, 1991.

Kwon, Y.S.; Namgoong, H.; Kim, J. H.; Cho, I. H.; Kim, M. D.; Eom, T. G. \& Koo , K. Effect of microthreads on removal torque and bone-to-implant contact: an experimental study in miniature pig. J. Periodontal Implant Sci., 43:41-6, 2013.

Oliveira, G. R.; Olate, S.; Cavalieri-Pereira, L.; Pozer, L.; Asprino, L. \& de Moraes, M. Maxillary sinus floor augmentation using blood without graft material. Preliminary results in 10 patients. J. Oral Maxillofac. Surg., 2013.

Wennerberg, A.; Albrektsson, T. \& Lausmac, J. Torque and histomophometric evaluation of cp titanium screw blasted with 25and $75 \mathrm{~mm}$-sized particles of Al2O3. J. Biomed. Mater. Res., 30:251-60, 1996.
Thomas, K. A.; Kay, J. F.; Cook, S. D. \& Jarcho, M. The effect of surface macrotexture and hydroxyapatite coating on the mechanical strengths and histologic profiles of titanium implant materials. J. Biomed. Mater. Res., 21(12):1395-414, 1987.

Rich, A. \& Harris, A. K. Anomalous preferences of cultured macrophages for hydrophobic and roughened substrata. J. Cell Sci., 50:1-7, 1981.

\author{
Dirección de Correspondencia \\ Prof. Dr. Sergio Olate \\ Unidad de Cirugía Oral y Maxilofacial \\ Universidad de La Frontera \\ Claro Solar No 115 \\ Temuco \\ CHILE
}

Teléfono: 56-45-2325000

Email: sergio.olate@ufrontera.cl

Recibido : 24-02-2013

Aceptado: 18-04-2013 